Vilma Santana 1

\title{
Acidentes de trabalho não fatais em adolescentes
}

\author{
Non-fatal work-related injuries \\ in adolescents
}

Martha Itaparica ${ }^{1}$

Andréa M. de Amorim 1

José Bouzas Araújo Filho 1

Gustavo Araújo 1

Mônia Oliveira 1

Sharon Cooper 2

\footnotetext{
1 Programa Integrado em Saúde Ambiental e do Trabalhador, Instituto de Saúde Coletiva, Universidade Federal da Bahia. Rua Padre Feijó 29, Salvador, $B A$ 40110-170, Brasil. vilma@ufba.br

2 School of Public Health, University of Texas at Houston. 1200 Hermann Pressler E543, Houston, TX 77030, U.S.A.
}

\begin{abstract}
This community-based survey estimates the annual incidence of non-fatal work-related injuries in adolescents and describes their characteristics. Using a random area sampling design, 2,513 households were selected from the city of Salvador, capital of Bahia State, Northeast Brazil. The study population included 361 young people 10 to 20 years of age who answered individual questionnaires on socio-demographic, occupational, and health data. Annual incidence of non-fatal work-related injuries was 6.4\%. Among females, most injuries were mild and were related to household chores; some 38.5\% required medical care, and $36.4 \%$ caused permanent non-disabling lesions. Among males, work-related injuries took place mainly in public byways, and the majority (60.0\%) were treated in emergency-care facilities. These results show that work-related injuries need to be considered in adolescent protection policies.
\end{abstract}

Key words Occupational Accidents; Adolescence; Occupational Health

Resumo Neste estudo estima-se a incidência anual e descrevem-se as características dos acidentes de trabalho não fatais em adolescentes com dados de um inquérito conduzido na cidade de Salvador, Bahia, Brasil. Utilizou-se amostra aleatória de superfície para selecionar 2.513 famílias, cujos membros foram entrevistados individualmente. A população do estudo compunhase de 361 jovens entre 10 e 20 anos. A incidência anual de acidentes de trabalho não fatais foi 6,4\%. Entre as mulheres, a maioria dos acidentes ocorreu no ambiente de trabalho doméstico $e$ tinha pequena gravidade, mas 38,5\% dos casos necessitaram de assistência médica, e 36,4\% deixaram seqüelas permanentes não incapacitantes. Entre os rapazes, os acidentes típicos na via pública predominaram, e a maioria (60,0\%) foi atendida em serviço de emergência. Esses resultados evidenciam que acidentes de trabalho entre jovens estão a merecer atenção nas políticas sociais no país.

Palavras-chave Acidentes de Trabalho; Adolescência; Saúde Ocupacional 


\section{Introdução}

A participação na força de trabalho de adolescentes é um problema social que vem se mantendo como uma questão ainda não resolvida em quase todo o mundo, tanto em países desenvolvidos como nos em desenvolvimento. A Organização Internacional do Trabalho (OIT), estima em mais de 120 milhões o número de crianças entre 5 e 14 anos de idade que trabalham em tempo integral, em países em desenvolvimento (Forastieri, 1997). Embora o Brasil represente a maior economia da América Latina, o trabalho de crianças e adolescentes ainda prevalece nas suas áreas menos industrializadas, particularmente no Nordeste (Brasil, 1999). Isso contrasta com as diretrizes do Conselho Nacional dos Direitos da Criança e Adolescentes (CONANDA), que definiu a erradicação do trabalho para menores de 14 anos (Brasil, 1995). Adolescentes, no entanto, podem celebrar contratos de trabalho, desde que em atividades não perigosas e condizentes com o seu desenvolvimento físico e psíquico (Brasil, 1999).

Estudos em outros países mostram que acidentes de trabalho são comuns entre adolescentes (Fassa et al., 2000; Graitcer \& Lerer, 1998; Runyan \& Zakocs, 2000). No Brasil, de acordo com os dados oficiais disponíveis, e portanto apenas para o trabalho legalizado ou formal, em 1997, foram registrados 4.314 benefícios concedidos em decorrência de acidentes de trabalho para menores de 18 anos de idade. A gravidade desse problema mostra-se evidente no registro de 218 óbitos entre homens e mulheres nessa faixa de idade como conseqüência deste tipo de acidente (Brasil, 2000). É provável ainda que esses dados estejam subestimados e sejam parciais, principalmente pela concentração de menores no setor informal da economia, ou pelo grande número de trabalhadores sem carteira, para os quais usualmente não se emitem registros administrativos desses eventos. Neste estudo, apresentam-se estimativas da incidência de acidentes de trabalho não fatais e suas características entre adolescentes de 10 a 20 anos, com base em dados de uma pesquisa realizada em Salvador, capital do Estado da Bahia.

\section{Métodos}

Os dados deste estudo provêm de um inquérito de base comunitária sobre trabalho e saúde, conduzido com uma amostra aleatória de superfície dessa área urbana, definida com um mapa aerofotográfico, com a ajuda do qual sorteou-se subáreas, onde se identificaram todas as famílias residentes. Estimaram-se cerca de 90 famílias em cada subárea. Como se esperava aproximadamente 3,8 pessoas por família (IBGE, 2000), calculou-se em 29 o número de subáreas necessário para se alcançar o tamanho da amostra desejado, de 2.500 famílias. A escolha desse desenho amostral deveu-se principalmente às características urbanas dessa cidade, onde são comuns a falta e a imprecisão de endereços, especialmente na periferia ou em bairros pobres, como também um acentuado crescimento e adensamento da população, dificilmente captados em mapas, além da necessidade de garantia de segurança aos pesquisadores de campo.

Pesquisadores treinados realizaram entrevistas individuais, que se iniciava com um levantamento de todos os membros da família, dos quais se registravam dados sócio-demográficos e ocupacionais para identificar a elegibilidade para responder a questionários específicos. Pessoas que tinham uma atividade remunerada forneciam dados da história ocupacional e de saúde. Caso relatassem a ocorrência de acidente nos últimos doze meses, período de referência do estudo, respondiam a perguntas específicas sobre esses eventos. Estas se iniciavam com uma narrativa sumária do acidente, e quando este se encontrava relacionado com o trabalho, seguiam-se questões sobre as suas características. Considerou-se como acidentes de trabalho, "qualquer dano infligido ao corpo por transferência de energia durante o trabalho (típico), ou no deslocamento trabalho/casa (trajeto) que envolvesse uma curta duração entre exposição e efeitos identificáveis, aproximadamente 48 horas após a ocorrência do evento/circunstância" (Hagberg et al., 1997:111). Desse modo, ao sujeito da pesquisa não se formulava nenhuma pergunta direta sobre "acidente de trabalho", considerando-se que nem sempre esses eventos são reconhecidos como tal, especialmente por jovens ou trabalhadores do setor informal da economia, que não dispõem de proteção legal para esses agravos. Quando havia referência a mais de um acidente no período do estudo, considerou-se nesta análise, apenas o mais recente. Características dos acidentes de trabalho foram: local e causa, tipo da lesão, atendimento médico, incapacidade de trabalhar após o acidente, efeitos do acidente sobre a capacidade de trabalho referido, e que foi classificada como: (1) sem efeito permanente completa recuperação da capacidade funcional e integridade física e psicológica após o acidente; (2) com efeito permanente, possibilitando trabalhar na mesma atividade; (3) com efeito permanente mas com capacidade apenas pa- 
ra exercer outro tipo de atividade; (4) efeito permanente com incapacidade para o trabalho, e a percepção sobre a prevenibilidade.

Devido à escassez de informações sobre as características do trabalho feito por adolescentes, e as conhecidas diferenças de sexo e idade, apresentam-se os dados descritivos sobre a participação na força de trabalho de acordo com estas variáveis. A idade foi tratada em três categorias: 10-14, 15-17 e 18-20 anos, enquanto o trabalho foi examinado para os seguintes grupos: sem trabalho - aqueles que referiram não ter atualmente atividade remunerada e que não participavam do trabalho doméstico para a família; trabalho apenas em casa - referiram ter responsabilidade pelo trabalho doméstico para a própria família em pelo menos oito horas por semana; trabalho remunerado - referiram alguma atividade paga; trabalho pago e em casa - acumulavam trabalho remunerado e afazeres domésticos para a família em pelo menos 8 horas por semana. Devido ao pequeno número de trabalhadores de 10 a 14 anos, considerou-se as faixas de 10-17 e 18-20 nas análises subseqüentes. As demais variáveis sóciodemográficas foram: cor da pele - identificada pelo entrevistador e classificada como negra (negros e mulatos) e não negra (brancos, índios e asiáticos); nível sócio-econômico - baseado no total dos bens da família ou equipamentos disponíveis no domicílio, que foi categorizado em baixo - menos de dois itens; médio - três a cinco itens e alto - mais que cinco itens; estudante atual (sim/não) e turno escolar, matutino, vespertino e noturno. As variáveis ocupacionais foram: vínculo/natureza de trabalho, setor da ocupação, jornada e local de trabalho, meio de transporte, treinamento recebido, carteira de trabalho assinada, e motivo para não ter carteira assinada.

A incidência anual foi calculada dividindose o número de casos identificados de acidentes de trabalho ocorridos no período de referência, pelo total da população em risco. Note que embora o estudo seja transversal, o fato de acidentes serem eventos circunscritos no tempo, permite a identificação de casos incidentes com base em informações referidas. As estimativas de incidência ajustadas para o desenho amostral, aleatório de superfície, foram calculadas empregando-se o Proc Surveymeans do SAS 8.11, pacote estatístico empregado para toda a análise. Inferências estatísticas para diferenças de proporções foram realizadas com o Teste do Qui Quadrado de Pearson. Para garantir a qualidade dos dados, reentrevistas parciais foram realizadas com $10 \%$ dos participantes além de dupla digitação por pessoas dife- rentes, utilizando-se o Epi Info 6.0. As bases de dados foram então comparadas e os dados checados e corrigidos. O protocolo da pesquisa foi submetido e aprovado por uma Comissão de Ética em Pesquisa da Universidade Federal da Bahia. Todo participante forneceu consentimento após o esclarecimento, mantido o anonimato e confidencialidade das informações, bem como todos os cuidados de proteção dos sujeitos. Toda a equipe da pesquisa foi instruída quanto aos dispositivos e responsabilidades éticas do estudo.

\section{Resultados}

Das 2.513 famílias selecionadas para o estudo, identificaram-se 9.591 pessoas, das quais 2.361 eram jovens na faixa de idade entre 10 e 20 anos $(24,6 \%)$, sendo que destes, $1.274(54,0 \%)$ eram do sexo feminino e 1.087 (46,0\%) do sexo masculino. O quadro geral da participação na força de trabalho é mostrado na Tabela 1, podendose verificar que $37,9 \%(n=894)$ eram trabalhadores que se concentravam no grupo de trabalho doméstico sem remuneração, para a própria família $(22,6 \%, n=533)$, enquanto $15,3 \%$ $(\mathrm{n}=361)$ desenvolviam alguma atividade remunerada. Em geral, não se observam grandes diferenças de gênero na participação no mercado de trabalho, exceção para o trabalho doméstico para a família e a dupla jornada de trabalho mais comuns entre as mulheres do que entre os homens. Ao contrário, enquanto 13,4\% dos indivíduos do sexo masculino têm apenas um trabalho pago, apenas $8,8 \%$ das mulheres encontram-se nessa posição.

Para o estudo da distribuição dos acidentes de trabalho não fatais, considerou-se como população para análise apenas os 382 trabalhadores remunerados, dos quais 21 (5,5\%) não responderam aos questionários individuais, restando 361 trabalhadores. As características sociodemográficas dessa população são mostradas na Tabela 2. A proporção de trabalhadores que freqüentavam a escola se reduz com a idade, diferença estatisticamente significante apenas entre pessoas do sexo masculino $(\mathrm{p}<0,05)$. Ao contrário, tanto entre as mulheres quanto entre os homens, a proporção de estudantes no turno noturno se eleva com a idade, diferenças estatisticamente significantes. Não houve diferenças de acordo com o gênero e idade para as demais variáveis em consideração.

Características ocupacionais dos adolescentes são apresentadas nas Tabelas 3 e 4 . Para o sexo feminino, nota-se que os vínculos empregatícios mais comuns foram como empregadas 
Tabela 1

Distribuição dos adolescentes no mercado de trabalho segundo o sexo e o grupo de idade $(n=2.361)$.

\begin{tabular}{|c|c|c|c|c|c|c|c|c|c|c|c|}
\hline \multirow{4}{*}{ Variáveis } & \multirow{4}{*}{$\begin{array}{c}\text { Total } \\
n=2.361\end{array}$} & \multicolumn{10}{|c|}{ Perfil de trabalho } \\
\hline & & & & & & \multicolumn{6}{|c|}{ Trabalho remunerado $(n=361)^{1}$} \\
\hline & & \multicolumn{2}{|c|}{ Não trabalham } & \multicolumn{2}{|c|}{ Apenas em casa } & \multicolumn{2}{|c|}{ Pago e em casa } & \multicolumn{2}{|c|}{ Apenas pago } & \multicolumn{2}{|c|}{ Subtotal } \\
\hline & & $n=1.467$ & $62,1 \%$ & $n=533$ & $22,6 \%$ & $n=103$ & $4,4 \%$ & $n=258$ & $10,9 \%$ & $n=361$ & $15,3 \%$ \\
\hline \multicolumn{12}{|l|}{ Mulheres } \\
\hline \multicolumn{12}{|l|}{ Idade } \\
\hline $10-14$ & 473 & 323 & 68,3 & 139 & 29,4 & 5 & 1,0 & 6 & 1,3 & 11 & 2,3 \\
\hline $15-17$ & 372 & 178 & 47,8 & 149 & 40,1 & 19 & 5,1 & 26 & 7,0 & 45 & 12,1 \\
\hline $18-20$ & 429 & 142 & 33,1 & 158 & 36,8 & 49 & 11,5 & 80 & 18,6 & 129 & 30,1 \\
\hline Subtotal & 1.274 & 643 & 50,5 & 446 & 35,0 & 73 & 5,7 & 112 & 8,8 & 185 & 14,5 \\
\hline \multicolumn{12}{|l|}{ Homens } \\
\hline \multicolumn{12}{|l|}{ Idade } \\
\hline $10-14$ & 441 & 399 & 90,5 & 30 & 6,8 & 3 & 0,7 & 9 & 2,0 & 12 & 2,7 \\
\hline $15-17$ & 315 & 232 & 73,6 & 33 & 10,5 & 7 & 2,2 & 43 & 13,7 & 50 & 15,9 \\
\hline $18-20$ & 331 & 193 & 58,3 & 24 & 7,3 & 20 & 6,0 & 94 & 28,4 & 114 & 34,4 \\
\hline Subtotal & 1.087 & 824 & 75,8 & 87 & 8,0 & 30 & 2,8 & 146 & 13,4 & 176 & 16,2 \\
\hline
\end{tabular}

1 Duas mulheres de 18 a 20 anos tinham dois trabalhos pagos. Um homem de 15 a 17 anos e três homens de 18 a 20 anos tinham 2 trabalhos pagos.

Tabela 2

Características sócio-demográficas dos adolescentes que referiram trabalho remunerado na população de estudo.

\begin{tabular}{|c|c|c|c|c|c|c|c|c|c|c|c|c|}
\hline \multirow[t]{3}{*}{ Variáveis } & \multicolumn{4}{|c|}{ Mulheres $(n=185)$} & \multicolumn{4}{|c|}{ Homens $(n=176)$} & \multicolumn{4}{|c|}{ Total $(\mathrm{n}=361)$} \\
\hline & \multicolumn{2}{|c|}{ 10-17 anos } & \multicolumn{2}{|c|}{$18-20$ anos } & \multicolumn{2}{|c|}{ 10-17 anos } & \multicolumn{2}{|c|}{$18-20$ anos } & \multicolumn{2}{|c|}{ 10-17 anos } & \multicolumn{2}{|c|}{$18-20$ anos } \\
\hline & $n=56$ & $\%$ & $n=129$ & $9 \%$ & $n=62$ & $\%$ & $n=114$ & $\%$ & $\mathrm{n}=118$ & $\%$ & $n=243$ & $\%$ \\
\hline \multicolumn{13}{|l|}{ Cor da pele } \\
\hline Negra & 37 & 66,1 & 81 & 62,8 & 42 & 67,7 & 69 & 60,5 & 79 & 66,9 & 150 & 61,7 \\
\hline Não negra & 19 & 33,9 & 48 & 37,2 & 20 & 32,3 & 45 & 39,5 & 39 & 33,1 & 93 & 38,3 \\
\hline \multicolumn{13}{|c|}{ Nível sócio-econômico } \\
\hline Baixo & 36 & 64,3 & 64 & 49,6 & 36 & 58,0 & 58 & 50,9 & 72 & 61,0 & 122 & 50,2 \\
\hline Médio & 14 & 25,0 & 45 & 34,9 & 22 & 35,5 & 36 & 31,6 & 36 & 30,5 & 81 & 33,3 \\
\hline Alto & 6 & 10,7 & 20 & 15,5 & 4 & 6,5 & 20 & 17,5 & 10 & 8,5 & 40 & 16,5 \\
\hline \multicolumn{13}{|c|}{ Estudante atual } \\
\hline Sim & 38 & 67,9 & 74 & 57,4 & 49 & 79,0 & 65 & $\star 57,5$ & 87 & 73,7 & 139 & 57,4 \\
\hline Não & 18 & 32,1 & 55 & 42,6 & 13 & 21,0 & 48 & 42,5 & 31 & 26,3 & 103 & 42,6 \\
\hline \multicolumn{13}{|l|}{ Turno escolar 1} \\
\hline Matutino & 18 & 48,7 & 11 & $\star \star 15,5$ & 19 & 38,8 & 10 & $\star 15,4$ & 37 & 43,0 & 21 & 18,4 \\
\hline Vespertino & 10 & 27,0 & 12 & 16,9 & 13 & 26,5 & 7 & 10,8 & 23 & 26,7 & 19 & 16,7 \\
\hline Noturno & 9 & 24,3 & 48 & 67,6 & 17 & 34,7 & 48 & 73,8 & 26 & 30,3 & 74 & 64,9 \\
\hline
\end{tabular}

Referente ao teste do qui quadrado.

${ }^{*} p<0,05$.

$\star * p<0,0001$

n = Total da população do estudo.

1 Total se refere apenas aos que estudam. Não se obtiveram informações de todos os estudantes. 
Características ocupacionais das adolescentes trabalhadoras remuneradas.

\begin{tabular}{|c|c|c|c|c|c|c|}
\hline \multirow[t]{3}{*}{ Variáveis } & \multicolumn{4}{|c|}{ Mulheres } & \multicolumn{2}{|c|}{ Total } \\
\hline & \multicolumn{2}{|c|}{ 10-17 anos } & \multicolumn{2}{|c|}{$18-20$ anos } & \\
\hline & $n=56$ & $\%$ & $n=129$ & $\%$ & $\mathrm{n}=185$ & $\%$ \\
\hline \multicolumn{7}{|l|}{ Vínculo $(n=184)$} \\
\hline Biscateira & 14 & 25,0 & 13 & 10,2 & 27 & 14,7 \\
\hline Autônoma & 4 & 7,1 & 9 & 7,0 & 13 & 7,1 \\
\hline Empregada doméstica1 & 18 & 32,2 & 42 & 32,8 & 60 & 32,6 \\
\hline Assalariada sem comissão & 12 & 21,4 & 40 & 31,2 & 52 & 28,3 \\
\hline Assalariada com comissão & 7 & 12,5 & 16 & 12,5 & 23 & 12,5 \\
\hline Outro & 1 & 1,8 & 8 & 6,3 & 9 & 4,8 \\
\hline \multicolumn{7}{|l|}{ Setor da ocupação $(n=164)$} \\
\hline Serviços gerais/domésticos & 20 & 43,5 & 46 & 39,0 & 66 & 40,2 \\
\hline Comércio & 18 & 39,1 & 29 & 24,6 & 47 & 28,7 \\
\hline Administração/ensino & 8 & 17,4 & 37 & 31,4 & 45 & 27,5 \\
\hline Indústria & 0 & - & 3 & 2,5 & 3 & 1,8 \\
\hline Transporte & 0 & - & 2 & 1,7 & 2 & 1,2 \\
\hline Segurança & 0 & - & 1 & 0,8 & 1 & 0,6 \\
\hline \multicolumn{7}{|l|}{ Jornada $(n=132)$} \\
\hline Comercial & 22 & 55,0 & 59 & 64,2 & 81 & 61,4 \\
\hline De turno & 10 & 25,0 & 23 & 25,0 & 33 & 25,0 \\
\hline Noturna & 5 & 12,5 & 5 & 5,4 & 10 & 7,6 \\
\hline Outra & 3 & 7,5 & 5 & 5,4 & 8 & 6,0 \\
\hline \multicolumn{7}{|l|}{ Local de trabalho $(n=133)$} \\
\hline Empresa/firma & 14 & 34,2 & 51 & 55,4 & 65 & 48,9 \\
\hline Na residência de terceiros & 9 & 21,9 & 13 & 14,2 & 22 & 16,5 \\
\hline Via pública & 8 & 19,5 & 8 & 8,7 & 16 & 12,0 \\
\hline Na própria residência & 5 & 12,2 & 7 & 7,6 & 13 & 9,0 \\
\hline Repartição pública & 3 & 7,3 & 6 & 6,5 & 9 & 6,8 \\
\hline Outros & 2 & 4,9 & 7 & 7,6 & 9 & 6,8 \\
\hline \multicolumn{7}{|l|}{ Transporte para o trabalho $(n=120)$} \\
\hline Ônibus & 16 & 45,7 & 51 & 60,0 & 67 & 55,8 \\
\hline Andando & 16 & 45,7 & 26 & 30,6 & 42 & 35,0 \\
\hline Carro próprio & 2 & 5,7 & 1 & 1,2 & 3 & 2,5 \\
\hline Ônibus empresa & 0 & - & 2 & 2,3 & 2 & 1,7 \\
\hline Carro empresa & 1 & 2,9 & 1 & 1,2 & 2 & 1,7 \\
\hline Outros & 0 & - & 4 & 4,7 & 4 & 3,3 \\
\hline Treinamento recebido $(n=183)$ & 14 & 25,5 & 49 & 38,3 & 63 & 34,4 \\
\hline \multicolumn{7}{|l|}{ Carteira assinada $(n=181)$} \\
\hline Sim & 4 & 7,3 & 36 & $\star * 28,6$ & 40 & 22,1 \\
\hline Não & 51 & 92,7 & 90 & 71,4 & 141 & 77,9 \\
\hline
\end{tabular}

(continua) 
Tabela 3 (continuação)

\begin{tabular}{|c|c|c|c|c|c|c|}
\hline \multirow[t]{3}{*}{ Variáveis } & \multicolumn{4}{|c|}{ Mulheres } & \multicolumn{2}{|c|}{ Total } \\
\hline & \multicolumn{2}{|c|}{ 10-17 anos } & \multicolumn{2}{|c|}{$18-20$ anos } & \multirow[b]{2}{*}{$\mathrm{n}=185$} & \multirow[b]{2}{*}{$\%$} \\
\hline & $n=56$ & $\%$ & $n=129$ & $\%$ & & \\
\hline \multicolumn{7}{|c|}{$\begin{array}{l}\text { Motivo de não ter } \\
\text { carteira de trabalho }(n=91)\end{array}$} \\
\hline Falta de oportunidade & 14 & 38,9 & 27 & 49,1 & 41 & 45,0 \\
\hline O patrão não quis & 4 & 11,1 & 12 & 21,8 & 16 & 17,6 \\
\hline Devido à idade & 7 & 19,4 & 0 & - & 7 & 7,7 \\
\hline Pouco estudo & 0 & - & 2 & 3,6 & 2 & 2,2 \\
\hline Nunca pensou & 2 & 5,6 & 0 & - & 2 & 2,2 \\
\hline Não tem patrão & 0 & - & 1 & 1,8 & 1 & 1,1 \\
\hline Outro & 9 & 25,0 & 13 & 23,7 & 22 & 24,2 \\
\hline
\end{tabular}

1 A ocupação de emprego em serviços domésticos foi considerada como um vínculo empregatício devido às particularidades deste serviço.

** $X^{2}=p<0,05 ; n=$ número da população do estudo.

domésticas $(32,6 \%)$ e assalariadas sem comissão (28,3\%). Para o sexo masculino, observou-se uma predominância de assalariados sem comissão (44,6\%) e biscateiros (26,9\%). O setor de ocupação que congrega a maior proporção de mulheres foi o de serviços gerais/domésticos $(40,2 \%)$ enquanto que para o sexo masculino, foi o comércio $(44,1 \%)$. Pessoas do sexo masculino se distribuem em maior número de setores de atividade quando comparados às mulheres. Estas, na faixa de 10 a 17 anos, trabalhavam mais comumente em casa de terceiros do que as de 18 a 20 anos. Entre os homens, a maior proporção de trabalhadores remunerados, independentemente da faixa de idade, trabalhava em empresas/firmas $(\mathrm{p}<0,05)$. Treinamento para o trabalho foi uma experiência relatada por $34,4 \%$ das mulheres e $52,3 \%$ dos homens, aumentando com a idade, diferença estatisticamente significante para o sexo masculino ( $p$ $<0,10)$. Quanto à formalidade das vinculações ocupacionais, observou-se que apenas $22,1 \%$ das mulheres e $26,1 \%$ dos homens tinham carteira assinada, que também aumenta com a idade, diferenças estatisticamente significantes para ambos os sexos. O principal motivo alegado para não possuir carteira assinada foi a falta de oportunidade, cujas freqüências foram estimadas em $45,0 \%$ para as mulheres e $42,4 \%$ para os homens, respectivamente.

Na Tabela 5, podem ser vistas as estimativas de incidência anual de acidentes de trabalho não fatais, que para a população geral foi estimada em $6,4 \%(23 / 359)$, maior entre as mulheres $(7,0 \%)$ do que entre os homens $(5,7 \%)$. Tanto para as mulheres quanto para os homens, verifica-se que a incidência diminuiu com a idade. Especificamente, entre as mulheres variou de $9,1 \%$ no grupo de 10 a 17 anos, para $6,3 \%$ entre as de mais idade, e respectivamente $8,1 \%$ e $4,4 \%$ entre os homens. Nenhuma dessas diferenças foi estatisticamente significante.

As características dos acidentes de trabalho não fatais ocorridos entre as mulheres são mostradas na Tabela 6. Para a totalidade desse grupo, observa-se que a maioria dos acidentes ocorreu na casa do patrão, sendo queda a principal causa referida, com maior freqüência nas de maior idade em comparação com as mais jovens. Queimadura foi o tipo de lesão mais comum. A maioria das mulheres acidentadas não referiu ter recebido atendimento médico, incapacidade para o trabalho, ou efeitos permanentes. Quanto à prevenibilidade, a maioria considerou que o acidente poderia ter sido evitado. Vale ressaltar que acidentes de trabalho mais graves parecem ocorrer mais comumente entre as mulheres com idade acima de 17 anos, quando comparadas ao grupo mais jovem. Dados semelhantes para as pessoas do sexo masculino são apresentados na Tabela 7. Verificase que o local do acidente variou largamente de acordo com a faixa etária. Por exemplo, entre os mais jovens os acidentes mais comuns foram os típicos, ocorridos na via pública, enquanto no grupo de mais idade, ao contrário, prevaleceram os acidentes de trajeto. Para as demais variáveis, não houve grandes diferenças com a idade, estimando-se que foram mais freqüentes os acidentes devido a quedas de veículos, sendo as lacerações o tipo de lesão mais comum. A gravidade desses acidentes de trabalho pode ser avaliada pela freqüência dos que buscaram atendimento médico e referiram ter ficado com incapacidade permanente, que alcançou $60,0 \%$, ressaltando-se um caso de acidente de trajeto sofrido por um adolescente de 19 anos que sofreu traumatismo craniano, fi- 
Características ocupacionais dos adolescentes trabalhadores remunerados.

\begin{tabular}{|c|c|c|c|c|c|c|}
\hline \multirow[t]{3}{*}{ Variáveis } & \multicolumn{4}{|c|}{ Homens } & \multicolumn{2}{|c|}{ Total } \\
\hline & \multicolumn{2}{|c|}{ 10-17 anos } & \multicolumn{2}{|c|}{$18-20$ anos } & & \\
\hline & $\mathrm{n}=62$ & $\%$ & $\mathrm{n}=114$ & $\%$ & $n=176$ & $\%$ \\
\hline \multicolumn{7}{|l|}{ Vínculo $(n=175)$} \\
\hline Biscateiro & 21 & 33,9 & 26 & 23,0 & 47 & 26,9 \\
\hline Autônomo & 11 & 17,7 & 8 & 7,1 & 19 & 10,9 \\
\hline Empregado doméstico 1 & 1 & 1,6 & 1 & 0,9 & 2 & 1,1 \\
\hline Assalariado sem comissão & 20 & 32,3 & 58 & 51,3 & 78 & 44,6 \\
\hline Assalariado com comissão & 4 & 6,5 & 12 & 10,6 & 16 & 9,1 \\
\hline Outro & 5 & 8,0 & 8 & 7,1 & 13 & 7,4 \\
\hline \multicolumn{7}{|l|}{ Setor da ocupação $(n=145)$} \\
\hline Comércio & 24 & 51,1 & 40 & 40,8 & 64 & 44,1 \\
\hline Administração/ensino & 10 & 21,3 & 18 & 18,4 & 28 & 19,3 \\
\hline Construção & 3 & 6,4 & 16 & 16,3 & 19 & 13,1 \\
\hline Indústria & 5 & 10,6 & 5 & 5,1 & 10 & 6,9 \\
\hline Serviços gerais/domésticos & 1 & 2,1 & 8 & 8,2 & 9 & 6,2 \\
\hline Outros & 4 & 8,4 & 11 & 11,2 & 15 & 10,4 \\
\hline \multicolumn{7}{|l|}{ Jornada $(n=174)$} \\
\hline Comercial & 40 & 65,6 & 76 & 67,3 & 116 & 66,7 \\
\hline De turno & 14 & 22,9 & 25 & 22,1 & 39 & 22,4 \\
\hline Noturna & 3 & 4,9 & 5 & 4,4 & 8 & 4,6 \\
\hline Outra & 4 & 6,6 & 7 & 6,2 & 11 & 6,3 \\
\hline \multicolumn{7}{|l|}{ Local de trabalho $(n=174)$} \\
\hline Empresa/firma & 25 & 41,0 & 70 & $* * 62,0$ & 95 & 54,6 \\
\hline Via pública & 20 & 32,8 & 11 & 9,7 & 31 & 17,8 \\
\hline Na residência de terceiros & 1 & 1,6 & 13 & 11,5 & 14 & 8,0 \\
\hline Na própria residência & 4 & 6,6 & 3 & 2,7 & 7 & 4,0 \\
\hline Repartição pública & 5 & 8,2 & 5 & 4,4 & 10 & 5,8 \\
\hline Outros & 6 & 9,8 & 11 & 9,7 & 17 & 9,8 \\
\hline \multicolumn{7}{|c|}{ Transporte para o trabalho $(n=165)$} \\
\hline Ônibus & 24 & 42,1 & 55 & $\star * 50,9$ & 79 & 47,9 \\
\hline Andando & 28 & 49,1 & 36 & 33,3 & 64 & 38,8 \\
\hline Outros & 5 & 8,8 & 17 & 15,8 & 22 & 13,3 \\
\hline Treinamento recebido $(n=176)$ & 26 & 41,9 & 66 & $\star 57,9$ & 92 & 52,3 \\
\hline \multicolumn{7}{|l|}{ Carteira assinada $(n=176)$} \\
\hline Sim & 3 & 4,8 & 43 & $\star \star \star 37,7$ & 46 & 26,1 \\
\hline Não & 59 & 95,2 & 71 & 62,3 & 130 & 73,9 \\
\hline \multicolumn{7}{|l|}{$\begin{array}{l}\text { Motivo de não ter carteira } \\
\text { de trabalho }(n=111)\end{array}$} \\
\hline Falta de oportunidade & 17 & 33,3 & 30 & $\star \star 50,0$ & 47 & 42,4 \\
\hline O patrão não quis & 4 & 7,8 & 13 & 21,6 & 17 & 15,3 \\
\hline Devido à idade & 14 & 27,5 & 1 & 1,7 & 15 & 13,5 \\
\hline Pouco estudo & 4 & 7,8 & 0 & - & 4 & 3,6 \\
\hline Não tem patrão & 0 & - & 4 & 6,7 & 4 & 3,6 \\
\hline Nunca pensou & 3 & 5,9 & 1 & 1,7 & 4 & 3,6 \\
\hline Deficiência física & 0 & - & 1 & 1,7 & 1 & 0,9 \\
\hline Outro & 9 & 17,7 & 10 & 16,6 & 19 & 17,1 \\
\hline
\end{tabular}

1 A ocupação de emprego em serviços domésticos foi considerada como um vínculo empregatício devido às particularidades deste serviço.

$X^{2}={ }^{*} p<0,10 ;{ }^{* \star} p<0,05 ;{ }^{\star \star \star} p<0,0001 ; n=$ número da população do estudo. 
Incidência bruta de acidentes de trabalho não fatais ${ }^{1}$ de acordo com o sexo e faixa etária.

\begin{tabular}{lccccccccc}
\hline Sexo & \multicolumn{1}{c}{ Faixa etária } & & \multicolumn{2}{c}{ Total } \\
& $\mathrm{N}=117$ & $\begin{array}{c}10-17 \text { anos } \\
\mathrm{n}=10\end{array}$ & $\mathrm{I}=8,5 \%$ & $\mathrm{~N}=242$ & $\mathrm{n}=13$ & $\mathrm{I}=5,4 \%$ & $\mathrm{~N}=359$ & $\mathrm{n}=23$ & $\mathrm{I}=6,45$ \\
\hline Feminino & 55 & 5 & 9,1 & 128 & 8 & 6,3 & 183 & 13 & 7,0 \\
Masculino & 62 & 5 & 8,1 & 114 & 5 & 4,4 & 176 & 10 & 5,7 \\
\hline
\end{tabular}

$\mathrm{N}$ = número da população do estudo; $\mathrm{n}$ = número de casos de acidentes de trabalho não fatais; $\mathrm{I}=$ incidência anual.

1 Nenhuma diferença foi estatisticamente significante.

cando com incapacidade para exercer qualquer tipo de trabalho. Apesar de dispor de carteira de trabalho assinada, não teve o acidente notificado, ficando sem os benefícios correspondentes.

\section{Discussão}

A comparação dos resultados deste estudo com os de outras investigações fica limitada porque acidentes de trabalho em adolescentes são raramente focalizados em epidemiologia. Até mesmo estudo sobre o trabalho de adolescentes ou crianças são escassos, inexistindo estatísticas confiáveis sobre a dimensão desse importante problema social, o que tem levado instituições a incluírem esse tema entre as suas diretrizes de políticas.

\section{O trabalho do adolescente - dimensão e características}

Com os dados deste estudo, verifica-se que o trabalho de adolescentes é comum, mesmo no contexto de um grande centro urbano que vem sendo alvo da campanha de erradicação do trabalho infantil, e cujo impacto positivo foi estimado pela redução de 4,4\% entre os adolescentes no mercado de trabalho entre 1988 e 1998 (Fernandes \& Mendonça, 1999). Mesmo considerando-se os limites legais impostos ao trabalho entre os menores de 14 anos de idade, ainda assim estimou-se que quase igual proporção de mulheres e homens, respectivamente $2,3 \%$ e $2,7 \%$, referiram desenvolver alguma atividade remunerada. Isso contrasta com o trabalho para a família, ao se verificar o grande diferencial por sexo, com um percentual de mulheres que realizam afazeres domésticos mais de quatro vezes maior $(29,4 / 6,8 \%)$ do que o de homens. Com base nos dados do Censo para o mesmo ano desta pesquisa, estima-se em 6.596 o número de crianças entre 10 e 14 anos de ida- de, que se encontra no mercado de trabalho na cidade de Salvador. Os dados existentes provêm em sua maioria da área rural, onde é comum o trabalho de crianças na agricultura, tanto para as mulheres quanto para os homens (Committee on the Health and Safety Implications of Child Labor, 1998; Fassa et al., 2000).

Ainda com os dados deste estudo, verificase que é a partir dos 14 anos de idade que a participação na força de trabalho de adolescentes se intensifica. Portanto, essa etapa da vida revela-se como fundamental para a entrada no mercado de trabalho, embora a esta época, adolescentes não tenham ainda alcançado a idade necessária para a conclusão dos estudos secundários. Nota-se que não houve diferenças estatisticamente significantes de gênero na participação no mercado de trabalho, em todas as faixas de idade, como já observado em outros estudos, quando se inclui o trabalho doméstico (Graitcer \& Lerer, 1998). Como era de se esperar, verificou-se que essa entrada precoce do adolescente no mercado de trabalho está possivelmente associada com o abandono escolar notadamente entre os rapazes, ou com a migração para o turno noturno, para ambos os sexos. Conforme encontrado em outros estudos (Fernandes \& Mendonça, 1999; Santana \& Almeida Filho, 1994), mulheres trabalhadoras tinham como ocupação mais comum o emprego em serviços domésticos ou serviços gerais, possivelmente pela sua natureza de extensão do trabalho que se desenvolve na família, e a não exigência de qualificação formal. A maior precariedade do trabalho desenvolvido pelas mulheres em comparação aos homens, fica evidente ao se verificar que elas recebem menos freqüentemente treinamento e têm menor proporção de vínculo formal de trabalho, em todas as faixas de idade consideradas. Os trabalhadores do sexo masculino se concentraram no setor de comércio, atividade muito comum nos grandes centros urbanos, mas apresentaram maior distribuição em outros setores, re- 
Características dos acidentes de trabalho entre adolescentes trabalhadoras remuneradas.

\begin{tabular}{|c|c|c|c|c|c|c|}
\hline \multirow[t]{3}{*}{ Variáveis } & \multicolumn{4}{|c|}{ Mulheres } & \multicolumn{2}{|c|}{ Total } \\
\hline & \multicolumn{2}{|c|}{ 10-17 anos } & \multicolumn{2}{|c|}{$18-20$ anos } & & \\
\hline & $n=5$ & $\%$ & $\mathrm{n}=8$ & $\%$ & $n=13$ & $\%$ \\
\hline \multicolumn{7}{|l|}{ Local do acidente } \\
\hline Trabalho na casa do patrão & 3 & 60,0 & 2 & 25,0 & 5 & 38,4 \\
\hline Trabalho em casa & 1 & 20,0 & 3 & 37,5 & 4 & 30,8 \\
\hline Estabelecimento/empresa & 0 & - & 1 & 12,5 & 1 & 7,7 \\
\hline Trabalho em via pública & 1 & 20,0 & 0 & - & 1 & 7,7 \\
\hline Trajeto & 0 & - & 1 & 12,5 & 1 & 7,7 \\
\hline Outro & 0 & - & 1 & 12,5 & 1 & 7,7 \\
\hline \multicolumn{7}{|l|}{ Causa do acidente } \\
\hline Queda & 1 & 20,0 & 5 & 71,4 & 6 & 50,0 \\
\hline Contato com substâncias quentes & 2 & 40,0 & 2 & 28,6 & 4 & 33,4 \\
\hline Atingido por veículo em movimento & 1 & 20,0 & 0 & - & 1 & 8,3 \\
\hline $\begin{array}{l}\text { Contato com superfícies } \\
\text { aquecidas/muito frias }\end{array}$ & 1 & 20,0 & 0 & - & 1 & 8,3 \\
\hline \multicolumn{7}{|l|}{ Tipo da lesão } \\
\hline Queimadura & 3 & 75,0 & 2 & 25,0 & 5 & 41,7 \\
\hline Hematoma & 0 & - & 2 & 25,0 & 2 & 16,7 \\
\hline Estiramento & 0 & - & 2 & 25,0 & 2 & 16,7 \\
\hline Raladura & 0 & - & 1 & 12,5 & 1 & 8,3 \\
\hline Laceração & 0 & - & 1 & 12,5 & 1 & 8,3 \\
\hline Outro & 1 & 25,0 & 0 & - & 1 & 8,3 \\
\hline \multicolumn{7}{|l|}{ Atendimento médico } \\
\hline Sim & 1 & 20,0 & 4 & 50,0 & 5 & 38,5 \\
\hline Não & 4 & 80,0 & 4 & 50,0 & 8 & 61,5 \\
\hline \multicolumn{7}{|l|}{$\begin{array}{l}\text { Incapacidade de trabalhar } \\
\text { após o acidente }\end{array}$} \\
\hline $\operatorname{Sim}$ & 0 & - & 4 & 50,0 & 4 & 30,8 \\
\hline Não & 5 & 100,0 & 4 & 50,0 & 9 & 69,2 \\
\hline \multicolumn{7}{|l|}{ Efeitos do acidente } \\
\hline Sem efeito permanente & 2 & 50,0 & 5 & 71,4 & 7 & 63,6 \\
\hline $\begin{array}{l}\text { Com efeito permanente, } \\
\text { sem incapacidade para } \\
\text { o trabalho na mesma atividade }\end{array}$ & 2 & 50,0 & 2 & 28,6 & 4 & 36,4 \\
\hline \multicolumn{7}{|l|}{ Prevenibilidade do acidente } \\
\hline $\operatorname{Sim}$ & 3 & 60,0 & 4 & 57,1 & 7 & 58,3 \\
\hline Não & 1 & 20,0 & 1 & 14,3 & 2 & 16,7 \\
\hline Não sabe & 1 & 20,0 & 2 & 28,6 & 3 & 25,0 \\
\hline
\end{tabular}

$\mathrm{n}=$ Total de acidentados. 
Características dos acidentes de trabalho entre adolescentes trabalhadores remunerados.

\begin{tabular}{|c|c|c|c|c|c|c|}
\hline \multirow[t]{3}{*}{ Variáveis } & \multicolumn{4}{|c|}{ Homens } & \multicolumn{2}{|c|}{ Total } \\
\hline & \multicolumn{2}{|c|}{ 10-17 anos } & \multicolumn{2}{|c|}{$18-20$ anos } & & \\
\hline & $\mathrm{n}=5$ & $\%$ & $n=5$ & $\%$ & $\mathrm{n}=10$ & $\%$ \\
\hline \multicolumn{7}{|l|}{ Local do acidente } \\
\hline Trabalho em via pública & 3 & 60,0 & 0 & - & 3 & 30,0 \\
\hline Trajeto & 0 & - & 2 & 40,0 & 2 & 20,0 \\
\hline Estabelecimento/empresa & 0 & - & 1 & 20,0 & 1 & 10,0 \\
\hline Firma terceirizada & 1 & 20,0 & 0 & - & 1 & 10,0 \\
\hline Trabalho em casa & 0 & - & 1 & 20,0 & 1 & 10,0 \\
\hline Trabalho na casa do patrão & 0 & - & 1 & 20,0 & 1 & 10,0 \\
\hline Outro & 1 & 20,0 & 0 & - & 1 & 10,0 \\
\hline \multicolumn{7}{|l|}{ Causa do acidente } \\
\hline Queda de veículo & 3 & 75,0 & 1 & 20,0 & 4 & 44,5 \\
\hline $\begin{array}{l}\text { Manejo de instrumentos } \\
\text { pérfuro-cortantes }\end{array}$ & 0 & - & 2 & 40,0 & 2 & 22,2 \\
\hline Atingido por veículo em movimento & 0 & - & 1 & 20,0 & 1 & 11,1 \\
\hline Colisão de veículo & 0 & - & 1 & 20,0 & 1 & 11,1 \\
\hline Contato com substâncias químicas & 1 & 25,0 & 0 & - & 1 & 11,1 \\
\hline \multicolumn{7}{|l|}{ Tipo da lesão } \\
\hline Laceração & 1 & 25,0 & 4 & 80,0 & 5 & 55,6 \\
\hline Raladura & 2 & 50,0 & 0 & - & 2 & 22,2 \\
\hline Hemorragia & 0 & - & 1 & 20,0 & 1 & 11,1 \\
\hline Outro & 1 & 25,0 & 0 & - & 1 & 11,1 \\
\hline \multicolumn{7}{|l|}{ Atendimento médico } \\
\hline $\operatorname{Sim}$ & 2 & 40,0 & 4 & 80,0 & 6 & 60,0 \\
\hline Não & 3 & 60,0 & 1 & 20,0 & 4 & 40,0 \\
\hline \multicolumn{7}{|l|}{$\begin{array}{l}\text { Incapacidade de trabalhar } \\
\text { após o acidente }\end{array}$} \\
\hline Sim & 3 & 60,0 & 3 & 60,0 & 6 & 60,0 \\
\hline Não & 2 & 40,0 & 2 & 40,0 & 4 & 40,0 \\
\hline \multicolumn{7}{|l|}{ Efeitos do acidente } \\
\hline Sem efeito permanente & 4 & 80,0 & 4 & 80,0 & 8 & 80,0 \\
\hline $\begin{array}{l}\text { Com efeito permanente, } \\
\text { sem incapacidade para } \\
\text { o trabalho na mesma atividade }\end{array}$ & 1 & 20,0 & 0 & - & 1 & 10,0 \\
\hline $\begin{array}{l}\text { Com efeito permanente, } \\
\text { com incapacidade para } \\
\text { qualquer tipo de trabalho }\end{array}$ & 0 & - & 1 & 20,0 & 1 & 10,0 \\
\hline \multicolumn{7}{|l|}{ Prevenibilidade do acidente } \\
\hline $\operatorname{Sim}$ & 2 & 40,0 & 3 & 60,0 & 5 & 50,0 \\
\hline Não & 2 & 40,0 & 2 & 40,0 & 4 & 40,0 \\
\hline Não sabe & 1 & 20,0 & 0 & - & 1 & 10,0 \\
\hline
\end{tabular}

$\mathrm{n}=$ total de acidentados. 
conhecidamente perigosos, como a construção civil, a indústria e o transporte, que juntos somavam cerca de $25,5 \%$ dos rapazes que trabalhavam. Além disso, observou-se também uma alta proporção de jovens (10 a 17 anos) cujo trabalho era realizado na via pública, local onde prevalecem não apenas riscos para a segurança física mas também para o consumo de drogas e a delinqüência (Graitcer \& Lerer, 1998).

\section{Acidentes de trabalho entre adolescentes}

A incidência anual de acidentes de trabalho não fatais foi alta $(6,4 \%)$, um pouco mais elevada entre as mulheres do que entre os homens, reduzindo-se com a idade em ambos os sexos. Esses dados são próximos aos encontrados em um estudo realizado com trabalhadores residentes em cidades de médio porte do interior do Estado de São Paulo, que empregou metodologia semelhante. Os autores estimaram igual incidência na faixa de 10 a 13 anos, que se reduziu para $4,1 \%$ no grupo de $14-17$, e chegou a $4,8 \%$ entre os de 18 a 24 anos, para um ano de referência (Barata et al., 2000). Maior freqüência anual de acidentes de trabalho não fatais $(18,9 \%)$ foi encontrada em adolescentes com menos de 16 anos de idade, trabalhadores da zona rural do Rio Grande do Sul (Fehlberg et al., 2001). Também utilizando dados obtidos com questionários aplicados a uma amostra de estudantes entre 10 e 20 anos, de escolas públicas do interior de São Paulo, verificou-se que $47,0 \%$ da população do estudo referiu ter sofrido acidentes de trabalho alguma vez na vida (Fischer et al., 2000). A comparação com resultados de estudos de outros países, para os quais alguns dados estão disponíveis, é limitada devido a diferenças na participação de crianças e adolescentes no mercado de trabalho.

É importante notar a escassez desses estudos e a ampla variação dos achados de acordo com a metodologia empregada (Runyan \& Zakocs, 2000). Por exemplo, nos Estados Unidos, com dados auto-referidos de uma amostra de estudantes do segundo grau, verificou-se que $17,0 \%$ referiram acidentes de trabalho não fatais no último ano (Parker et al., 1994). Esse achado se encontra muito acima da taxa anual de 9,0\% estimada para adolescentes entre $16 \mathrm{e}$ 17 anos, com base em registros de pedidos de compensação (Miller \& Kaufmann, 1998), ou com dados de serviços de emergência, respectivamente, $4,7 \%$ entre os de 15 anos, $5,5 \%$ nos de 16 e 5,3\% para trabalhadores de 17 anos de idade (Brooks et al., 1993). Tendência à redução com a idade da taxa de adolescentes acidentados que haviam recebido compensação, tam- bém foi encontrada por Belville et al. (1993), mas aparentemente os seus dados limitavamse a casos mais graves. Os dados deste estudo mostram uma tendência inversa com a idade, que tem sido explicada pela inadequação das ferramentas e equipamentos de trabalho às dimensões corporais de crianças e adolescentes, à falta de treinamento, e também pelo amadurecimento psicomotor e cognitivo (Fassa et al., 2000; Forastieri, 1997). Além disso, como em muitos contextos, o trabalho feito por crianças é ilegal, as condições de trabalho per se são mais insalubres e menos sujeitas à vigilância $\mathrm{e}$ medidas de proteção do que aqueles onde predominam adultos (Committee on the Health and Safety Implication of Child Labor, 1998; Cooper et al., 1999).

Neste estudo não se verificam as diferenças de gênero encontradas por outros autores, de maior incidência de acidentes de trabalho entre os homens em relação às mulheres (Belville et al., 1993; Layne et al., 1994; Miller \& Kaufman, 1998), mesmo nas pesquisas realizadas no Brasil (Fischer et al., 2000). Todavia, estes achados guardam consonância com os resultados de outras investigações (Evensen et al., 2000; Fehlberg et al., 2001). É possível que as discordâncias desses achados residam nas faixas de idade em comparação, desde que a razão homem/mulher varia de acordo com a idade, e esses estudos incluíam distintas faixas etárias. Outras razões seriam o tipo e a origem dos dados, uma vez que acidentes de trabalho são mais graves entre os homens, esses certamente estariam mais representados nas bases de dados originados de serviços de saúde ou de sistemas de compensação para acidentes, ou ainda, naquelas que privilegiam ou se limitam a empregados formais ou da indústria, que concentram pessoas do sexo masculino. Como é um inquérito populacional, os achados deste estudo, por um lado, não estão afetados significativamente por esse viés. Mas por outro, como as mulheres são conhecidas por perceberem e referirem mais comumente eventos adversos de saúde, é possível que estas tenham referido mais comumente acidente devido à conhecida maior rememoração de eventos de saúde por parte do sexo feminino. Todavia, não se pode deixar de reconhecer que a maior freqüência de atividades domésticas entre as mulheres, nas quais os riscos para acidentes são comuns (Cayless, 2001; Hamzaoglu et al., 2002), pode ser uma explicação real para a pequena diferença por gênero na incidência dos acidentes de trabalho não fatais.

Como era de se esperar, dadas às diferenças ocupacionais de acordo com o sexo, houve 
também distinções relativas às características dos acidentes de trabalho. Por exemplo, enquanto entre as mulheres as causas principais foram as quedas, as queimaduras foram as lesões mais comuns, ambas possivelmente relacionadas com o trabalho em atividades domésticas. Entre os homens, prevaleceram as quedas de veículos, sendo a laceração a lesão mais freqüente, o que aponta para a falta de segurança no transporte ou no trabalho realizado em veículos, atividades que incorporam mais comumente pessoas do sexo masculino, o que foi também encontrado por Evensen et al. (2000). Consistentemente com a literatura (Runyan \& Zakocs, 2000), neste estudo, os acidentes de trabalho não fatais foram mais graves entre os homens do que entre as mulheres, considerandose que homens mais freqüentemente receberam atendimento médico ou referiram mais comumente incapacidade para o trabalho quando comparados às mulheres. Isso tem sido explicado pela maior concentração masculina em atividades que requerem o uso de ferramentas ou equipamentos perigosos, como na agricultura, indústria ou na construção civil (Evensen et al., 2000). Todavia, maior proporção de mulheres referiu ter se ausentado do trabalho devido ao acidente do que os homens, o que pode refletir a maior flexibilidade e aceitabilidade dos patrões, ou pelo tipo de trabalho, para a ausência das mulheres.

Os achados deste estudo devem ser considerados com o cuidado que requer estimativas de morbidade baseadas em dados referidos. Como se sustentam na rememoração de eventos, estão afetados por vieses negativos ou de omissão, que pode se originar tanto nos seus aspectos desfavoráveis; por exemplo, o sentimento de falha por não ter cumprido adequadamente uma tarefa, o estigma que pode levar ao afastamento do trabalho, dificuldades de obter um novo emprego e o sofrimento relacionado às lesões físicas ou psicológicas, e também a aspectos positivos como a pouca gravidade e o não reconhecimento de sua natureza ocupacional. Isso pode estar exacerbado entre jovens adolescentes, especialmente os do sexo masculino, que costumam minimizar a gravidade dos problemas de saúde, ou evitar a sua menção, movidos pelo sentimento de onipotência e invulnerabilidade típicos dessa fase da vida. Entre as mulheres, estes vieses também podem estar ocorrendo, mas para ambos os sexos não houve indícios da sua ocorrência ou de que estivessem produzindo distorções significativas nos resultados. A proximidade dos achados com os obtidos em outros estudos que empregaram metodologia semelhante é uma boa indicação da qualidade dos dados e adequação da abordagem utilizada. Todavia, deve-se apontar para o número insuficiente da população do estudo para a precisão de freqüências baixas como as encontradas, o que dificultou a interpretação das características dos acidentes de trabalho. Vale lembrar, todavia, que não foi propósito da pesquisa original o exame exclusivo de adolescentes trabalhadores. Este caráter secundário do presente estudo pode ter originado problemas, como a inadequação da linguagem utilizada nos instrumentos ou o uso de questionários para a coleta de dados. Estes, podem ter privilegiado os adultos, maioria da população estudada, e não terem sido os mais apropriados para os adolescentes que integravam o grupo aqui examinado.

Apesar desses limites, este é um dos raros estudos brasileiros que focalizam a freqüência anual de acidentes de trabalho em adolescentes, que tem como base a população geral, e certamente os seus achados contribuirão para uma melhor compreensão do problema, definição e efetivação de políticas de proteção e de prevenção de acidentes de trabalho para o trabalhador jovem. A invisibilidade dos acidentes de trabalho entre adolescentes surpreende, pois sequer são mencionados como um dos componentes importantes dos agravos à saúde devido a causas externas ou violência, reconhecidos como um dos principais problemas de saúde pública nos centros urbanos brasileiros. Vale mencionar os aspectos positivos do Programa de Erradicação do Trabalho Infantil (PETI) e do Programa Bolsa-Escola, que em grande parte do estado vêm sendo reconhecidos como iniciativas de sucesso no propósito de erradicar o trabalho feito por crianças. Todavia, isso não deve encobrir a necessária atenção requerida pelos adolescentes, também vulneráveis aos efeitos adversos do trabalho realizado em más condições, ou que podem comprometer a freqüência, a permanência e o desempenho escolar, hoje amplamente reconhecido como fundamentais para o desenvolvimento econômico e social do país. 


\section{Agradecimentos}

Este trabalho somente se tornou possível com a excepcional dedicação de todos os membros da nossa extensa equipe, e da população de Salvador que nos acolheu com carinho e respeito. É a ela que dedicamos esta pesquisa, esperando que possa vir a frutificar em melhores políticas de proteção para os trabalhadores.

Financiamento: Conselho Nacional de Desenvolvimento Científico e Tecnológico (Projeto Nordeste de Pesquisa, Proc. 521226/98-8) e Secretaria de Planejamento e Tecnologia do Estado da Bahia, Centro de Apoio ao Desenvolvimento Científico e Tecnológico. Apoio da University of North Carolina at Chapel Hill e da University of Texas at Houston, Estados Unidos.

\section{Referências}

BARATA, R. C. B.; RIBEIRO, M. C. S. A. \& MORAES, J. C., 2000. Acidentes de trabalho referidos por trabalhadores moradores em área urbana no interior do Estado de São Paulo. Informe Epidemiológico do SUS, 9:199-210.

BELVILLE, R.; POLLACK, S. H.; GODBOLD, J. H. \& LANDRIGAN, P. J., 1993. Occupational injuries among working adolescents in New York State. JAMA, 269:2754-2759.

BRASIL, 1995. Diretrizes Nacionais para a Política de Atenção à Infância e Adolescência. Brasília: Conselho Nacional dos Direitos da Criança e do Adolescente, Ministério da Justiça.

BRASIL, 1999. Estatuto da Criança e do Adolescente. 3 Março $2000<$ http://www.mtb.gov.br/esta/ acidentes $97 />$.

BRASIL, 2000. Trabalho Infantil - 2000. 3 Março 2000 $<$ http://www.mtb.gov.br/esta/acidentes97/>.

BROOKS, D. R.; DAVIS, K. L. \& GALLAGHER, S. S., 1993. Work-related injuries among Massachusetts children: A study based on emergency departments. American Journal of Industrial Medicine, 24:313-324.

CAYLESS, S. M., 2001. Slip, trip and fall accidents: Relationship to building features and use of coroners' reports in ascribing cause. Applied Ergonomics, 32:155-162.

COMMITTEE ON THE HEALTH AND SAFETY IMPLICATIONS OF CHILD LABOR, 1998. Protecting Youth at Work. Washington, DC: National Academy Press.

COOPER, S.; BURAU, K. D.; ROBISON, T. B.; RICHARDSIN, S.; SCHNITZER, P. G. \& FRASER Jr., J. J., 1999. Adolescent occupational injuries: Texas, 1990-1996. American Journal of Industrial Medicine, 35:43-50.
EVENSEN, C. T.; SCHULMAN, M. D.; RUNYAN, C. W.; ZAKOCS, C. W. \& DUNN, K. A., 2000. The downside of adolescent employment: Hazards and injuries among working teens in North Carolina. Journal of Adolescence, 23:545-560.

FASSA, A. C. G.; FACCHINI, L. A.; DALL'AGNOL, M. M \& CHRISTIANI, D. C., 2000. Child labor and health. International Journal of Occupational and Environmental Health, 6:55-62.

FEHLBERG, M. F.; SANTOS, I. \& TOMASI, E., 2001. Prevalência e fatores associados a acidentes de trabalho em zona rural. Revista de Saúde Pública, 35:269-275.

FERNANDES, M. C. \& MENDONÇA, L. K., 1999. O trabalho da criança e do adolescente na RMS. Bahia Análise \& Dados, 8:73-88.

FISCHER, F.; TEIXEIRA, L. R.; LATORE, M. R. D. O.; MARTINS, I. S.; OLIVEIRA, D. C.; COSTA, L. A. R.; MARINHO, S. P.; PERESTRELO, J. P. P. \& COOPER, S. P., 2000. Acidentes de Trabalho entre Estudantes do Ensino Fundamental e Médio das Cidades de Santo Antônio do Pinhal e Monteiro Lobato, SP. São Paulo: Faculdade de Saúde Pública, Universidade de São Paulo.

FORASTIERI, V., 1997. Children at Work: Health and Safety Risks. Geneva: International Labour Organization.

GRAITCER, P. L. \& LERER L. B., 1998. Child Labor and Health: Quantifying the Global Health Impacts of Child Labor. Washington, DC: World Bank.

HAGBERG, M.; CHRISTIANI, T. K.; COURTNEY, H. W.; LEAMON, T. B. \& SMITH, T. J., 1997. Conceptual and definitional issues in occupational injury epidemiology. American Journal of Industrial Medicine, 32:106-115.

HAMZAOGLU, O.; ÖZKAN, O. \& JANSON, S., 2002. In- 
cidence and causes of home accidents at Ankara Çigiltepe apartments in Turkey. Accident Analysis and Prevention, 34:123-128.

IBGE (Fundação Instituto Brasileiro de Geografia e Estatística), 2000. Dados do Censo 1991. 3 Março $2000<$ http://www.ibge.net/home/estatística/ população/>.

LAYNE, L. A.; CASTILLO, D. N.; STOUT, N. \& CUTLIP, P., 1994. Adolescent occupational injuries requiring hospital emergency departments. American Journal of Public Health, 84:657-660.

MILLER, M. E. \& KAUFMAN, J. D., 1998. Occupational injuries among adolescents in Washington State, 1988-1991. American Journal of Industrial Medicine, 34:121-138.
MTE (Ministério do Trabalho e Emprego), 2000. Estatísticas sobre Acidentes de Trabalho. Brasília: MTE.

PARKER, D. L.; CARL, W. R.; FRENCH, L. R. \& MARTIN, F. B., 1994. Characteristics of adolescent work injuries reported to the Minnesota Department of Labor and Industry. American Journal of Public Health, 84:606-610.

RUNYAN, C. W. \& ZAKOCS, R. C., 2000. Epidemiology and prevention of injuries among adolescent workers in the United States. Annual Review of Public Health, 21:247-269.

SANTANA, V. \& ALMEIDA FILHO, N., 1994. Emprego doméstico e sofrimento mental. Saúde Mental em Revista, 2:9-28.

Recebido em 4 de fevereiro de 2002

Versão final reapresentada em 29 de julho de 2002

Aprovado em 8 de outubro de 2002 